\title{
The Use of Exponential Smoothing (ES), Holts and Winter (HW) and ARIMA Models in electricity Price Analysis
}

\author{
$\underline{\text { Yasir Alsaedi }}^{\text {a b (D) and Gurudeo Anand Tularam b }}$ (i) \\ ${ }^{a}$ Department of Mathematics, Umm Al-Qura University, Makkah, Saudi Arabia, ${ }^{b}$ Centre for Planetary \\ Health and Food Security, Griffith University, Australia \\ Email: yasir.alsaedi@griffithuni.edu.au
}

\begin{abstract}
Electricity pricing is among the most important contemporary policy issues facing Australia, and electricity prices represent a critical component of on-going discussions concerning energy and climate change policies. In fact, several attempts to move forward with such policies have been stymied by concerns about potential increases in electricity prices. One example of the crisis in action can be seen in the closure of the Hazelwood Power Station, which slashed Victoria's energy supply by $22 \%$, causing annual energy bills to soar by an average of $\$ 300$ per household. Therefore, energy analysts and government organizations alike require guidelines to help them choose the most appropriate forecasting techniques so that they can achieve accurate predictions of spot pricing trends.

The aim of the present study is to compare the application of the exponential smoothing (ES), Holt-Winters (HW), and autoregressive integrated moving average (ARIMA) forecasting methods in relation to spot electricity pricing. The assessment is made in the context of the Australian National Electricity Market (ANEM). In order to determine the most appropriate model, four different strategies are applied as selection criteria in order to quantify the accuracy of the model predictions, namely the mean squared error (MSE), rootmean-square error (RMSE), mean absolute error (MAE) and mean absolute percentage error (MAPE) strategies. The comparison indicates that the HW model performs better than the ES model in terms of its predictive power, with a confidence interval of $95 \%$. However, the ARIMA $(1,1,1)$ model yielded the best results, leading us to conclude that this sophisticated and robust model outperformed other simple yet flexible models in electricity market. This study will make two significant contributions to the current literature. First, it will help policymakers and industry marketing strategists to select the most appropriate forecasting method for the spot electricity market. Second, it will help traders to assess the appropriate position at over-the-counter and well-developed futures trading platforms (e.g., ASX), since the accurate forecasting of spot prices is important for derivatives pricing.
\end{abstract}

Keywords: $\quad$ Spot electricity price, Forecasting, time series analysis 
Alsaedi \& Tularam, The Use of Exponential Smoothing (ES), Holts and Winter (HW) and ARIMA Models in electricity Price Analysis

\section{INTRODUCTION}

Forecasting involves the prediction or estimation of an actual value during some future time period or for another situation (Tularam and Ilahee, 2010). It is concerned with what the future will look like, not with what it should look like. Forecasting models capable of predicting future events are used in numerous practical fields, including business, economics, finance, science, and engineering (Alsaedi et al., 2019; Alsaedi and Tularam, 2020). Further, different models have been used to forecast data over particular periods (Alsaedi et al., 2020a, b). There are various methods available for the forecasting of energy prices, including statistical time series models (Tularam and Saeed, 2016), artificial neural networks (Panapakidis and Dagoumas, 2016), wavelet transform models (Voronin and Partanen, 2014), as well as ensemble and portfolio decision models (Neupane et al., 2017). However, within the current literature, the time series model appears to be the most popular forecasting method due to its ability to deal with non-stationarity, seasonal patterns, and signals (Alsaedi et al., 2019).

Two types of time series forecasting models are available, namely the univariate and multivariate models. Univariate time series forecasting involves the use of a continuous variable to predict future values based on previously observed values, while multivariate models feature more parameters than univariate models. In forecasting, as well as in economics, it has often been observed that univariate methods can provide a more accurate forecast than multivariate methods (Abdel-Aal, 2008). The ES, HW, and ARIMA techniques are among the various methods that apply univariate time series.

Researchers have compared different models so as to examine the appropriateness of the forecasted data over given periods of time. This line of research has been partly motivated by the dynamic nature of the problem and partly by the need to achieve better results (Rahman and Ahmar, 2017; Tularam and Saeed, 2016). For example, Tularam and Saeed (2016) applied the ES, HW, and ARIMA techniques using MSE, RMSE, MAE, MAPE, and Theil's U-statistic criteria in order to compare the different models' oil price forecasting performance. Their results showed that the ARIMA model provided more accurate forecasts than the ES and HW models. Further, Rahman and Ahmar (2017) compared the ARIMA and HW models with regard to the prediction of total primary energy consumption data in the United States. Their results demonstrated that the HW model provided better results than the ARIMA model. Additionally, Voronin and Partanen (2014) combined wavelet transforms, ARIMA models, and neural networks to predict electricity prices in the Finnish energy market, which is part of the Nordic Power Pool. Their study concluded that ARIMA models are extremely important for both modelling electricity demand and price forecasting.

After reviewing the prior literature, it became clear that the univariate time series model is commonly used for studying trends and, further, that it offers the ability to forecast electricity prices. The present study focuses on comparing different univariate time series models with regard to the forecasting of spot pricing in the Australian electricity markets. Three types of univariate models are considered, namely the ES, HW, and ARIMA models. To determine the most appropriate model in this context, four different strategies are applied as selection criteria to quantify the accuracy of the models' predictions, namely the MSE, RMSE, MAE, and MAPE strategies.

The remainder of the paper is organized as follows. Section 2 describes the data source, methodology, and means of quantifying the forecasting accuracy. Section 3 outlines the empirical results of the study, while section 4 offers some concluding remarks.

\section{MATERIALS AND METHODS}

The average monthly spot electricity prices for the five market regions within the ANEM, namely New South Wales (NSW), Queensland (QLD), South Australia (SA), Victoria (VIC), and Tasmania (TAS) served as the central time series used in the present study. The datasets consisted of monthly data collected from the AEMO covers the period from January 2006 to December 2020. This time series was chosen because the fluctuating nature of the data endows it with extreme nonlinearity, which means that chaos might pose challenges in forecasting future prices. This study focused on an in-sample period, which means that the time series between January 2006 and December 2020 was used to generate the three models' forecasts, while the time series between January 2021 and December 2020 served as out-of-sample data against which the accuracies of the various forecasts were measured. The accuracies of the three models' spot electricity pricing (as expressed in Australian dollars per megawatt hour [\$/MWh]) forecasts were determined and interrelated based on four 
Alsaedi \& Tularam, The Use of Exponential Smoothing (ES), Holts and Winter (HW) and ARIMA Models in electricity Price Analysis

metrics, namely the MSE, RMSE, MAE, and MAPE strategies. Figure 1 shows the average monthly reported price values for the spot electricity prices in ANEM, as expressed in Australian dollars per MWh, for each month from January 2006 to December 2020.

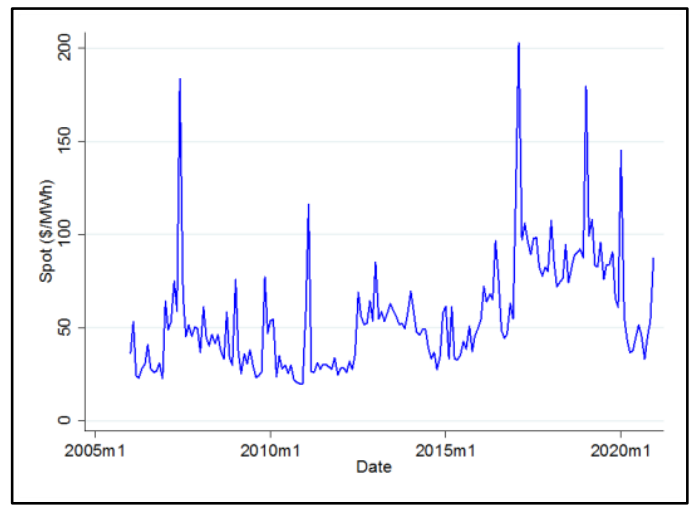

Figure 1. Australian spot electricity pricing time series

The following subsections present the different models used in the present study, both mathematically and theoretically. The mathematical expressions and applications of the four metrics used to quantify the forecast accuracies are also discussed.

\subsection{Exponential smoothing model}

The ES method involves smoothing out random fluctuations in time series data. Typically, it is used when the data pattern is approximately horizontal (i.e., there is neither cyclic variation nor a pronounced trend in the historical data). The ES equation is given as:

$$
L_{t}=\alpha Y_{t}+(1-\alpha) L_{t-1},
$$

where $L_{t}$ is the exponential smoothed value, $Y_{t}$ is the actual value during the time period $\mathrm{t}, \alpha$ is the unknown smoothing constant to be determined with a value between zero and one, and $L_{t-1}$ is the forecast or smoothed value for period $t$.

\subsection{Holt-Winters model}

The HW model is an extension of the ES model. It uses a linear combination of the previous values of a series to generate and model future values. It is applied to time series data that exhibits a trend. Hence, recent time series recordings are key to forecasting the future values of a given series. The model for the time series data $L_{t}$ is as shown below:

$$
\begin{gathered}
L_{t}=\alpha \frac{Y_{t}}{S_{t-M}}+(1-\alpha)\left(L_{t-1}+T_{t-1}\right) \\
T_{t}=\beta\left(L_{t}-L_{t-1}\right)+(1-\beta) T_{t-1} \\
S_{t}=\gamma \frac{Y_{t}}{L_{t}}+(1-\gamma) S_{t-M}
\end{gathered}
$$

where $L_{t}$ is the smoothed value, $\alpha$ is the smoothing constant $(0<\alpha<1), \beta$ is the trend smoothing constant $(0<\beta<1), S_{t}$ is the seasonality estimate, $\gamma$ is the smoothing parameter for the seasonality estimate $(0<\gamma<$ 1 ), and $Y_{t}$ is the actual value during time period $\mathrm{t}$. 
Alsaedi \& Tularam, The Use of Exponential Smoothing (ES), Holts and Winter (HW) and ARIMA Models in electricity Price Analysis

\subsection{Autoregressive integrated moving average model}

The ARIMA model represents another common time series model that is very popular among data scientists. While the above-mentioned exponential smoothing models are based on a description of the trend and seasonality within the data, the ARIMA model aims to describe the correlations within the data. The ARIMA model is specified by three order parameters, namely $(\mathrm{p}, \mathrm{d}, \mathrm{q})$, where $\mathrm{p}$ indicates the order of the autoregressive part, $d$ the amount of the difference, and $q$ the order of the moving average part. The ARMA models is given as:

$$
\emptyset_{t}^{\wedge}=\sum_{i=1}^{p} \theta_{i} \emptyset_{t-i}+\varepsilon_{t}+\sum_{i=1}^{q} \emptyset_{i} \varepsilon_{t-i}
$$

where $\theta_{t}$ is the autoregression parameter at time $\mathrm{t}, \varepsilon_{t}$ is the error term at time $\mathrm{t}$, and $\emptyset_{t}$ is the moving average parameter at time $t$.

\subsection{Model selection criteria}

Error measures are used to compare how well a given model fits the relevant time series. As noted above, a number of selection criteria (i.e., metrics), namely the MSE, RMSE, MAE, and MAPE strategies, are used in this regard in the present study. According to Tularam and Saeed (2016), the best fit, or best forecasting model, is the one with only minimal errors. Table 1 illustrates how each criterion is calculated. The MSE is the first evaluation criterion to be utilized. The MSE represents the average squared difference between the estimated values and what is being estimated. It is almost always strictly positive (and not zero) due to randomness or because the estimator does not account for information that could produce a more accurate estimate. The second evaluation criterion, the RMSE, refers to the standard deviation of the residuals (i.e., the prediction errors). This approach is more sensitive, and it also minimizes large errors. Nevertheless, the ability to compare different time series is limited when using this second criterion.

Table 1. Model accuracy metrics.

\begin{tabular}{cccc}
\hline Criterion & Formula & Criterion & Formula \\
\hline MSE & $\frac{1}{n} \sum_{i=1}^{n} \varepsilon_{i}^{2}$ & RMSE & $\sqrt{M S E}$ \\
MAE & $\frac{1}{n} \sum_{i=1}^{n}\left|\varepsilon_{i}\right|$ & MAPE & $\frac{1}{n} \sum_{i=1}^{n}\left(\left|\frac{\varepsilon_{i}}{x_{i}}\right|\right) \times 100$ \\
\hline
\end{tabular}

When comparing the forecasting methods applied to a single time series, or to several time series with the same units, the third evaluation criterion, the MAE, is a popular approach, since it is easy to understand and compute. A forecasting method that minimizes the MAE will lead to forecasts of the median, while minimizing the RMSE will lead to forecasts of the mean. Consequently, the RMSE is also widely used, despite being more difficult to interpret. The MAE is the mean of the absolute errors. An absolute error is the absolute value of the difference between the forecasted value and the actual value. The MAE shows "how big an error" is, on average, expected from a given forecast. The major limitation of this approach is the fact that the relative size of the error is not always obvious. Sometimes, it is difficult to tell a longer error from a small error. To combat this problem, we can identify the mean absolute error in percentage terms (i.e., the MAPE). The MAPE, that is, the fourth evaluation criterion, enables the comparison of forecasts of different series in different scales. 
Alsaedi \& Tularam, The Use of Exponential Smoothing (ES), Holts and Winter (HW) and ARIMA Models in electricity Price Analysis

\section{RESULTS AND DISCUSSION}

In the present study, the ES, HW, and ARIMA models were used to forecast ANEM monthly electricity spot pricing from January 2006 to December 2020. The ES, HW, and ARIMA models were all implemented in Stata, which contains built-in functions that allow the user to spontaneously determine a model's parameters. The only requirement for the program is the time series data to be analysed.

Forecasting using the ES method requires the initialization of the smoothing parameter. ES is dependent on just one parameter $(\alpha)$ to estimate the level at any point in a given time series, with that parameter $(\alpha)$ having values between zero and one. Table 2 illustrates the results of the ES model of the spot electricity pricing in ANEM. Using Stata, the ES model indicates that the parameter $\alpha=0.320$ is the best parameter value. The equation for this model, therefore, takes the form:

$$
L_{t}=0.320 Y_{t}+0.68 L_{t-1}
$$

Figure 2 demonstrates the accuracy of the ES model. The black line indicates the $95 \%$ confidence interval, the solid blue line is the actual value, and the red dashed line is the forecast value. It can be seen that the results of the ES model do not satisfactorily predict the actual data. Compared with the ES model forecasts, those of the HW model are relatively accurate. The performance of the HW model relies heavily on the choice of parameters, as shown in Figure 3. The parameter values used to produce Figure 3 were $\alpha=0.6, \beta=0.3$ and $\gamma=0.1$. The forecast presented in Figure 3 is better than that of the ES model: the forecast prices fluctuate close to the actual prices. The actual prices also consistently fall well within the $95 \%$ confidence interval of the forecast from the time-series data. These results demonstrate that the HW model provides more accurate predictions than the sophisticated ES model.

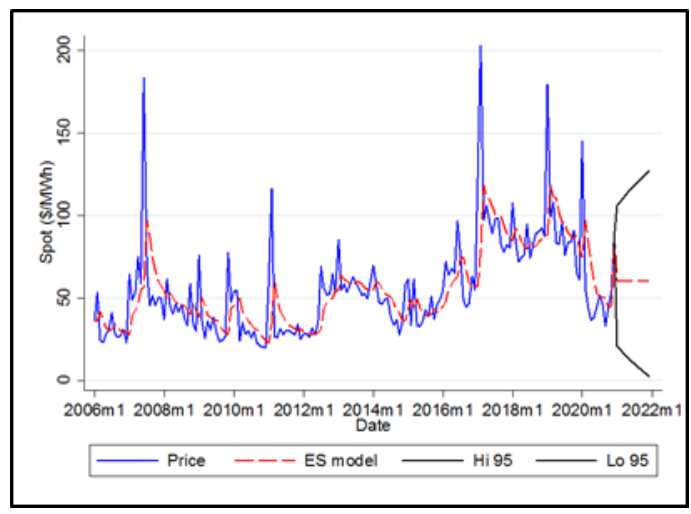

Figure 2. Results of the ES model

We found that the ARIMA $(1,1,1)$ model was the best-fitting model with respect to the spot electricity pricing data. The ARIMA $(1,1,1)$ model equation can be written as:

$$
\text { spot }_{t}=0.4390+0.3980 \text { spot }_{t-1}-0.9544 \varepsilon_{t-1}+\varepsilon_{t}
$$

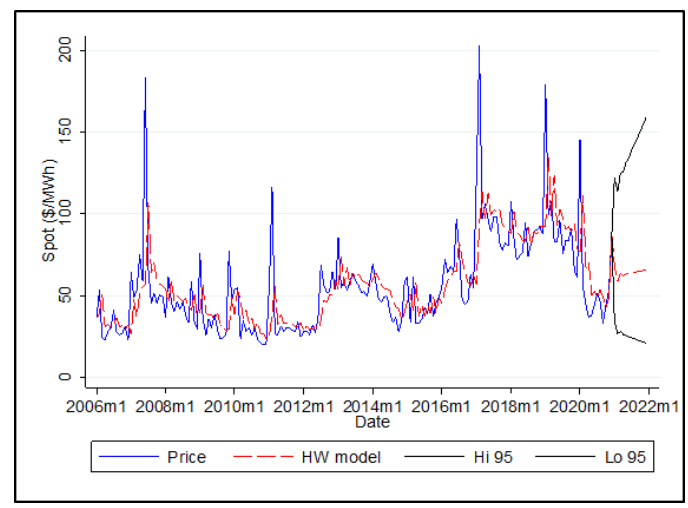

Figure 3. Results of the HW model 
Alsaedi \& Tularam, The Use of Exponential Smoothing (ES), Holts and Winter (HW) and ARIMA Models in electricity Price Analysis

Figure 4 offers a graphical representation of the values forecasted using the ARIMA method. It should be noted, however, that the ARIMA model is a fairly sophisticated model in terms of accurate forecasting. Figure 4, together with the ARIMA forecasts, shows that the $\operatorname{ARIMA}(1,1,1)$ results fit the actual time series data "considerably better" than those of the two previous models. The ARIMA model selects the parameters using a significance level of $5 \%$.

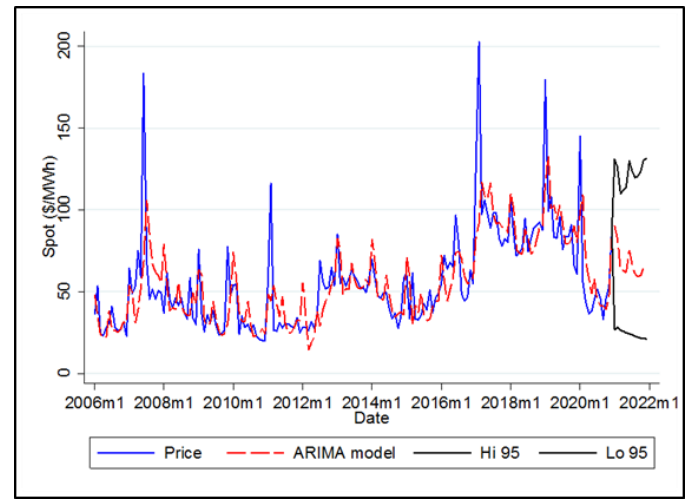

Figure 4. Results of the ARIMA model

The preceding subsections detailed the results of the three time series models that were used in this study to generate forecasts of the spot electricity prices in Australia. Every prediction was compared to the actual value of the respective time series of the spot electricity prices. The accuracy of each forecast was evaluated using four metrics, as discussed above. All four approaches were applied to determine and rank the performances of the models for the given time series.

Table 2 summarizes the three models and their respective forecasting performances. The MSE, RMSE, MAE, and MAPE approaches are all commonly used measures of the adequacy of forecasts. The rule of thumb holds that the smaller the MSE, RMSE, MAE, and MAPE results, the better the forecasting ability of the model. The present results thus demonstrate that the ARIMA model performs better than either of the other two models for the given time series. Indeed, the ARIMA $(1,1,1)$ model exhibited smaller prediction errors than the other two models, and so it was rightfully concluded that the ARIMA $(1,1,1)$ model represents the best model fit for the spot electricity pricing data. Importantly, although the HW model exhibited the second best forecast following that of the ARIMA model, the performance of each model relies on the utilized data.

Table 2. Measures of the errors of the three models (ES, HW, and ARIMA).

\begin{tabular}{cccc}
\hline $\begin{array}{c}\text { Measures of } \\
\text { accuracy }\end{array}$ & ES & HW & ARIMA \\
\hline MSE & 541.95 & 516.78 & $425.06^{*}$ \\
\hline RMSE & 23.280 & 22.733 & $20.617^{*}$ \\
\hline MAE & 14.099 & 14.036 & $12.718^{*}$ \\
\hline MAPE & 25.283 & 24.908 & $22.770^{*}$ \\
\hline
\end{tabular}

Despite all three models being black boxes, the differences observed between their performances are related to the differences that exist between the applied methods of determining forecasts in the ES and HW models as well as in the ARIMA model. The forecasting method used in the ES and HW models relies on a weighted average of the prior observed values, with the weights declining exponentially, which basically implies that the data for more recent observations contribute more significantly than previous data. The ARIMA model, however, involves three parts, namely the autoregression, integration, and moving average parts, with the future value of a given variable being a linear combination of the past values and the associated errors. 
Alsaedi \& Tularam, The Use of Exponential Smoothing (ES), Holts and Winter (HW) and ARIMA Models in electricity Price Analysis

\section{CONCLUSION}

This study examined the forecasting accuracies of three time series models that are simple to use and understand in relation to spot electricity pricing data. The models were the ES, HW, and ARIMA models. The results showed that the ARIMA $(1,1,1)$ model offered the best fit for the spot electricity pricing data, since its errors were smaller than those of the ES and HW models. Therefore, the ARIMA model could prove useful with regard to the electricity market when quick and cost-effective modelling is required. This finding may help policymakers and industry marketing strategists to select the most appropriate forecasting method for the spot electricity market. This finding is in line with the findings of other studies (Tularam and Saeed, 2016; Voronin and Partanen, 2014). The results obtained by Voronin and Partanen (2014) showed that ARIMA models are extremely important in electricity price forecasting compared to the various time-series methods. While predicting future events based on an appropriate time series model can aid in understanding the modelling process, we could further extend this work by examining and comparing more complicated univariate models, such as the autoregressive conditional heteroscedasticity model ( $\mathrm{ARCH})$, the generalized ARCH model (GARCH), and the ARIMA/GARCH model (Contreras, 2017).

Forecasting is vital when advising policy makers. Perfect decision making can only be achieved when changes are viewed from two perspectives, namely current events and what is likely to occur in the future. However uncertain a given forecast might appear, policy makers are compelled to consider its validity during their decision-making process. Ideally, policy makers would base their decisions on accurate forecasts in order to both strengthen their policies and achieve ultimate outcomes that differ from those forecasted. Therefore, the organizations and entities whose main activities rely on the spot, options, solar and wind electricity markets find such forecasts especially useful in terms of formulating their policies and marketing strategies so as to adequately adapt to the future changes forecasted by the models. When the utilized forecasts are accurate, the entities affiliated with electricity, solar and wind production can use the resultant information to make prudent decisions regarding the prices they attach to electricity, solar and wind power. As a result, through accurate knowledge of the flow of public money, as well as of the regular patterns seen in electricity, solar and wind and demand, they can modify spot, options, solar and wind prices in such a way as to avoid damaging their organizations' financial capability, or otherwise affecting organizational objectives.

\section{REFERENCES}

Abdel-Aal, R., 2008. Univariate modeling and forecasting of monthly energy demand time series using abductive and neural networks. Computers \& Industrial Engineering 54(4) 903-917.

Alsaedi, Y., Tularam, G.A., Wong, V., 2019. Application of ARIMA Modelling for the Forecasting of Solar, Wind, Spot and Options Electricity Prices: The Australian National Electricity Market. International Journal of Energy Economics and Policy 9(4) 263-272.

Alsaedi, Y., Tularam, G.A., Wong, V., 2020a. Assessing the Effects of Solar and Wind Prices on the Australia Electricity Spot and Options Markets Using a Vector Autoregression Analysis. International Journal of Energy Economics and Policy 10(1) 120-133.

Alsaedi, Y., Tularam, G.A., Wong, V., 2020b. Impact of Solar and Wind Prices on the Integrated Global Electricity Spot and Options Markets: A Time Series Analysis. International Journal of Energy Economics and Policy 10(2) 337-353.

Alsaedi, Y.H., Tularam, G.A., 2020. The relationship between electricity consumption, peak load and GDP in Saudi Arabia: A VAR analysis. Mathematics and Computers in Simulation 175 164-178.

Contreras, J., 2017. Forecasting models of electricity prices. Multidisciplinary Digital Publishing Institute.

Neupane, B., Woon, W.L., Aung, Z., 2017. Ensemble prediction model with expert selection for electricity price forecasting. Energies 10(1) 77.

Panapakidis, I.P., Dagoumas, A.S., 2016. Day-ahead electricity price forecasting via the application of artificial neural network based models. Applied Energy 172 132-151.

Rahman, A., Ahmar, A.S., 2017. Forecasting of primary energy consumption data in the United States: A comparison between ARIMA and Holter-Winters models, AIP Conference Proceedings. AIP Publishing, p. 020163.

Tularam, G.A., Ilahee, M., 2010. Time series analysis of rainfall and temperature interactions in coastal catchments. Journal of mathematics and statistics 6(3) 372-380.

Tularam, G.A., Saeed, T., 2016. Oil-price forecasting based on various univariate time-series models. American Journal of Operations Research 6(03) 226.

Voronin, S., Partanen, J., 2014. Forecasting electricity price and demand using a hybrid approach based on wavelet transform, ARIMA and neural networks. International Journal of Energy Research 38(5) 626-637. 